\title{
Investigación cualitativa en salud. Una revisión crítica de la producción bibliográfica en México
}

Francisco J Mercado-Martínez, M en MS, D en CS,(1) Blanca A Díaz, Lic en Psic,(1) Luz María Tejada-Tabayas, D en CSP, (2) Carlos D Ascencio-Mera, Lic en Psic. ${ }^{\left({ }^{(1)}\right.}$

\author{
Mercado-Martínez FJ, Díaz BA, \\ Tejada-Tabayas LM, Ascencio-Mera CD. \\ Investigación cualitativa en salud. Una revisión crítica \\ de la producción bibliográfica en México. \\ Salud Publica Mex 20 I ;53:504-5 I 2.
}

\section{Resumen}

Objetivo. Examinar críticamente la producción bibliográfica sobre la investigación cualitativa en salud (ICS) en México. Material y métodos. Revisión de 128 trabajos publicados en la década del 2000 al 2009. Su búsqueda y recuperación se hizo mediante consulta de índices, buscadores y bases de datos, así como consultando a académicos. Se hizo análisis de frecuencias y de contenido. Resultados. La producción en este campo se incrementa notoriamente en el periodo analizado, se difunde a nivel nacional e internacional y aparece mayoritariamente en revistas de salud pública y medicina social. Su consolidación, no obstante, dista de ser realidad dada su concentración institucional, territorial y temática, su dependencia de la teoría fundamentada, su olvido de temas sanitarios y actores importantes y su impacto marginal en el campo científico. Conclusiones. La investigación cualitativa en salud logra avances en México pero enfrenta serios desafíos para consolidarse. Varias propuestas se presentan para fortalecerla.

Palabras clave: investigación cualitativa; salud; revisión; México
Mercado-Martínez FJ, Díaz BA, Tejada-Tabayas LM, Ascencio-Mera CD.

Qualitative health research. A critical review of recent work in Mexico.

Salud Publica Mex 20I I;53:504-5 I 2.

\begin{abstract}
Objective. This paper critically examines recent work on qualitative health research (QHR) in Mexico. Material and Methods. A review was conducted of I 28 articles published between 2000 and 2009. A literature search was done drawing together a verse of indexes, search engine, data bases and citations, as well as interviewing researchers. A frequency and content analysis was carried out. Results. QHR has expanded significantly, both nationally and internationally, and papers published in public health and social medicine journals. However, several factors impede its consolidation: the institutional, territorial and thematic concentration of studies, the dependency on grounded theory, the selective nature of the research topics and populations; and its marginal scientific impact. Conclusions. QHR has grown in México, but its consolidation faces serious challenges. Several proposals are discussed that could strengthen the field.
\end{abstract}

Keywords: qualitative research; health; review; Mexico

(I) Departamento de Salud Pública. Centro Universitario de Ciencias de la Salud. Universidad de Guadalajara. Guadalajara, México

(2) Facultad de Enfermería. Universidad Autónoma de San Luis Potosí. San Luis Potosí, México

Fecha de recibido: 28 abril de 201 I - Fecha de aceptado: 2 I de septiembre de 201 I Autor de correspondencia: Dr. Francisco J. Mercado-Martínez. Jaime Nuno 1669-7, Col. Chapultepec Country. Guadalajara, México. Correo electrónico: Francisco.mercado@cucs.udg.mx 
L a investigación cualitativa (IC) ha tenido un incremento notorio a la par de una aceptación creciente en diversas áreas y disciplinas en Iberoamérica. ${ }^{1} \mathrm{Nu}$ merosos autores se han ocupado de documentar los avances logrados, sobre todo durante los primeros años de la década pasada. Entre otros, han dado cuenta de la producción generada en el campo de la salud, sea en el conjunto de la región, ${ }^{2}$ en varios países ${ }^{3}$ o en uno de ellos en particular, ${ }^{4}$ centrándose en temáticas sanitarias determinadas como la salud mental ${ }^{5} \mathrm{o}$ en los trabajos publicados en revistas específicas. ${ }^{6}$

México ha tenido una rica tradición en la investigación cualitativa en salud (ICS). ${ }^{7}$ Sin embargo, durante varios años no se han realizado evaluaciones sistemáticas de la producción generada en su conjunto. En consecuencia, hoy en día no se dispone de elementos que permitan examinar, reflexionar y comprender los logros, problemas y desafíos que presenta este campo en el país. Ello ha contribuido al desarrollo limitado de criterios que permitan valorar la calidad de la ICS; tampoco se han identificado los avances y obstáculos enfrentados así como las estrategias a implementar para fortalecerlo, formar recursos humanos y utilizar sus resultados en la esfera pública u otros ámbitos de la sociedad.

Por lo anterior, este trabajo se propone revisar críticamente la IC generada en el área de la salud en México. En particular, es de interés documentar tanto los avances como los desafíos que enfrenta la producción científica publicada durante una década.

\section{Material y métodos}

Se llevó a cabo una revisión crítica en gran parte de la producción bibliográfica sobre la IC en salud en México. Los trabajos fueron seleccionados según los criterios siguientes: 1) aquellos artículos empíricos cuya metodología fuese de corte cualitativo o cuali-cuantitativo;2) los trabajos teóricos o revisiones sobre la ICS; 3) publicados entre los años 2000 y 2009; 4) que hayan aparecido en revistas incluidas en el Índice de Revistas Mexicanas de Investigación del CONACYT, en PubMed o en bases de datos internacionales; ello con el fin de delimitar la búsqueda a las revistas arbitradas y asegurar un mínimo de calidad y pertinencia; 5) cuya temática se centrase en la salud-enfermedad-atención y; 6) cuyo primer autor estuviese adscrito a una institución mexicana, independientemente de su nacionalidad.

La búsqueda de los trabajos se realizó en medios electrónicos (índices, buscadores y bases de datos), consultando a académicos y revisando las citas. La revisión automatizada incluyó el índice PubMed, el buscador Google Académico y las bases de datos: Scielo,
Redalyc, ScienceDirect, EBSCO, OVID y Sage Complete. Se utilizaron grupos de palabras clave, tanto en español como en inglés, tales como investigación cualitativa y sus sinónimos, y de los enfoques teóricos y las metodologías más empleadas, como etnografía, fenomenología y teoría fundamentada; salud, enfermedad y padecimiento, así como descriptores de enfermedades específicas por ejemplo diabetes, adicciones y obesidad, así como México.

Elaboramos una lista de los autores encontrados hasta ese momento, los cuales fueron 90 de 28 instituciones. Se les envió un mensaje por correo electrónico a 70 de ellos -al ser imposible contactar a los 20 restantes- para informarles de la finalidad de nuestro trabajo de revisión y solicitarles sus publicaciones en el campo. Respondieron 38 de ellos y enviaron 108 trabajos. Paralelamente se revisaron las citas de los trabajos recuperados para detectar publicaciones que pudiesen ser incluidas según los criterios establecidos.

Se identificaron un total de 279 referencias, de las cuales se recuperaron 277 textos completos. Una vez revisados, se seleccionaron 128 trabajos que cumplieron los criterios de inclusión mencionados. 117 fueron estudios empíricos, cinco teóricos y seis revisiones.

La revisión, clasificación y síntesis de los 128 trabajos seleccionados se realizó mediante la elaboración de una matriz que incluía: a) datos del primer autor: sexo e institución de adscripción, b) Información sintética de los trabajos: objetivo, perspectiva teórica, metodología (diseño, población, lugar, tipo de muestra, obtención de la información, software empleado y análisis), resultados, propuestas y referencias bibliográficas. También se incluyeron datos de la revista: su factor de impacto (FI), el año y país de la publicación. El número de citas se obtuvo por medio de Google Académico al considerarlo como un recurso aceptable para el análisis de las citas. ${ }^{8}$ Finalmente, se llevó a cabo análisis de frecuencias y de contenido. ${ }^{9}$

\section{Resultados}

Las publicaciones de corte cualitativa en el área de la salud en México se incrementaron y difundieron progresivamente durante la década analizada. Del año 2000 al 2002 aparecieron cinco trabajos al año en promedio, aumentando la cifra a partir del 2003 hasta ascender a 24 en 2008; esto es casi cinco veces más que al inicio del decenio. Tales trabajos fueron publicados en 42 revistas de diversas áreas o disciplinas, pero el número mayor (70\%) lo fue en las de salud pública/medicina social/ salud colectiva; luego en las de ciencias sociales y humanidades (25\%), y en menor grado de psicología 
y psiquiatría (5\%). Escasos trabajos aparecieron en las del área clínica.

Se trata de una producción cada vez más difundida a nivel internacional, por lo menos en los países sede de las revistas empleadas. Los 128 trabajos analizados se publicaron en 10 países, tanto de América como de Europa. Además de México, aparecen en orden progresivo Brasil, Estados Unidos, Reino Unido, Colombia, España, Chile, Alemania, Argentina y Canadá. Varios de ellos se publicaron en revistas con reconocimiento académico internacional, tal como aquéllas cuyo FI es superior a 2.000. Este es el caso de aquellos artículos que aparecieron en las revistas AIDS, Social Science and Medicine, Qualitative Health Research, Journal of Health Communication y BMC Public Health.

Es notorio el número creciente de publicaciones en este campo, sin embargo, la mayoría de los 87 primeros autores encontrados estaban adscritos a pocas instituciones. Como se observa en el cuadro I, 79\% de los trabajos correspondía a investigadores de seis instituciones educativas de tres ciudades del centro occidente de México, principalmente del Instituto Nacional de Salud Pública (INSP), la Universidad de Guadalajara (UdeG), el Instituto Mexicano del Seguro Social (IMSS), el Instituto Nacional de Psiquiatría Ramón de la Fuente (INPRF), la Universidad Nacional Autónoma de México (UNAM) y la Universidad Autónoma Metropolitana (UAM). Mientras que 21\% restantes eran de autores adscritos a 15 instituciones del país, sobre todo educativas.

Otro tipo de centralización se constata dado el número reducido de países y revistas en donde aparecen estos trabajos. Visto en su conjunto, $85 \%$ de ellos fueron publicados en revistas de cuatro países: $52 \%$ mexicanas, $15 \%$ brasileñas, $10 \%$ norteamericanas y $9 \%$ del Reino Unido. Íntimamente relacionado con lo anterior, 2/3 del total de los artículos aparecieron en ocho revistas de esos mismos países, 28\% en Salud Pública de México,

\section{Cuadro I}

INSTITUCIÓN DE ADSCRIPCIÓN DEL PRIMER AUTOR DE los estudios cualitativos. MéXico, 2000-2009

\begin{tabular}{lcc} 
Institución & No. & $\%$ \\
INSP & 40 & 31.3 \\
\hline UdeG & 18 & 14.0 \\
\hline IMSS & 13 & 10.2 \\
\hline INPRF & 13 & 10.2 \\
\hline UNAM & 9 & 7.0 \\
\hline UAM & 8 & 6.3 \\
\hline Otras & 27 & 21.0 \\
\hline Total & 128 & 100.0
\end{tabular}

13\% en Salud Mental, una cifra similar (5\%) en las cuatro siguientes: Cadernos de Saúde Publica, Revista de Saude Publica, Qualitative Health Research e Investigación en Salud, y otro 4\% en la Revista Latino-Americana de Enfermagem. Los trabajos restantes aparecieron en otras 35 revistas. El $75 \%$ de los trabajos se publicaron en español, incluyendo aquellos que aparecieron en las revistas brasileñas y 25\% restante en inglés. Dos datos adicionales se orientan en la misma dirección. Las mujeres son las primeras autoras en $68 \%$ de los trabajos; por otro lado, $55 \%$ de los trabajos corresponden a investigadores adscritos a la misma institución, otro $29 \%$ implicó la colaboración de académicos de dos o más instituciones nacionales y sólo 16\% fueron realizados con investigadores de instituciones extranjeras.

Ocho temas sanitarios son objeto de atención en los estudios cualitativos analizados. Según se puede apreciar en el cuadro II, casi $85 \%$ de los trabajos examinan cinco de estos temas; $20 \%$ se refieren a las enfermedades crónicas, otro $20 \%$ a la salud sexual y reproductiva, $14 \%$ a las adicciones, $12 \%$ a la atención a la salud y $9 \%$ a la salud mental. Examinando los estudios con más detenimiento, los aspectos analizados tratan del mundo de las percepciones, las representaciones, los significados y las prácticas en torno a diversas condiciones mórbidas o los modelos de atención a la salud-enfermedad.

No obstante el aumento en la producción científica revisada en este campo, omisiones importantes se encuentran en ciertos temas sanitarios y grupos de la población. Más de la mitad de los estudios seleccionados (52\%) se realizan en dos ciudades del país, México y Guadalajara, lo cual deja un gran vacío en cuanto a lo que ocurre en otras ciudades y zonas del país. Aunado a lo anterior, $88 \%$ de los trabajos empíricos prestan atención a la población urbana, ocupándose sobre todo de las condiciones de los adultos del sector popular, los obreros y en menor medida la clase media. En el extremo opuesto, son inexistentes los estudios sobre la clase alta, y pocos se preocupan por los sectores rurales, los campesinos y los indígenas, así como de los niños, los jóvenes y los ancianos.

La mayoría de los estudios empíricos se adscriben teórica y conceptualmente a ciertas vertientes anglosajonas sobre cómo hacer IC, particularmente a la americana. Ello se constata al revisar los enfoques, las metodologías, las estrategias y las referencias empleadas. De entrada, $2 / 3$ de los trabajos no adoptan postura alguna o se adscriben a la teoría fundamentada (TF). En cambio, sólo un tercio adopta una postura teórica definida: $9 \%$ la teoría de las representaciones sociales, 5\% la antropología cognitiva, 4\% la fenomenología y unos cuantos la teoría crítica. La mayoría de los trabajos sustenta sus posturas y estrategias a partir de las obras de autores norteame- 
Cuadro II

Temas generales, porcentaje y PRoyectos seleccionados de los estudios cualitativos. México, 2000-2009

\begin{tabular}{|c|c|c|}
\hline Tema & $\%$ & Proyectos \\
\hline Salud sexual y reproductiva & 21.3 & $\begin{array}{l}\text { Significados y prácticas de quienes padecen } \mathrm{VIH}^{10} \\
\text { Significados y vulnerabilidad ante el } \mathrm{VIH} \text { en mujeres indígenas y varones migrantes } \\
\text { Percepciones de las mujeres y enfrentamiento del aborto } \\
\text { Representaciones sociales sobre el } \mathrm{VIH}^{13}\end{array}$ \\
\hline Enfermedades crónicas & 20.6 & $\begin{array}{l}\text { Experiencias del padecimiento }{ }^{14} \\
\text { Creencias y prácticas de pacientes con diabetes mellitus } \\
\text { Perspectivas y prácticas de personas con dolor crónico } \\
\text { Representaciones socioculturales y trayectorias de autoatención de personas con diabetes mellitus }{ }^{17}\end{array}$ \\
\hline Adicciones & 13.7 & $\begin{array}{l}\text { Representaciones sociales de los padres }{ }^{18} \\
\text { Percepciones y prácticas de personas con alcoholismo }{ }^{19} \\
\text { Percepciones de la población }{ }^{20}\end{array}$ \\
\hline Atención a la salud & 12.0 & $\begin{array}{l}\text { Estrategias de atención a la salud de los migrantes }{ }^{21} \\
\text { Relación médico-paciente y adherencia terapéutica en personas con } \mathrm{VIH}^{22} \\
\text { Percepciones de la atención a la salud } \\
\text { Calidad de la relación del personal de salud con los pacientes } \\
\text { Relación médico paciente }\end{array}$ \\
\hline Salud mental & 9.4 & $\begin{array}{l}\text { Representaciones sociales sobre la salud mental }{ }^{26} \\
\text { Prácticas de autocuidado para aliviar malestares emocionales }{ }^{27}\end{array}$ \\
\hline Nutrición & 7.7 & $\begin{array}{l}\text { Experiencias de las personas con obesidad }{ }^{28} \\
\text { Percepciones del cuerpo y prácticas alimentarias de las mujeres }{ }^{29} \\
\text { Consenso cultural de las adolescentes embarazadas sobre su alimentación }{ }^{30}\end{array}$ \\
\hline Violencia & 6.8 & $\begin{array}{l}\text { Experiencias de violencia en mujeres embarazadas }{ }^{31} \\
\text { Significados y prácticas de profesionales de salud sobre la violencia doméstica }{ }^{32} \\
\text { Significado de las adolescentes sobre la violencia sexual }{ }^{33}\end{array}$ \\
\hline Cuidado de la salud & 5.1 & Conocimientos y prácticas de las mujeres ${ }^{34}$ \\
\hline Otros & 19.0 & $\begin{array}{l}\text { Percepciones del gremio médico en el marco de la descentralización del sector de salud } \\
\text { Experiencias de la salud y enfermedad en el envejecimiento }{ }^{36}\end{array}$ \\
\hline Total & $115.6^{*}$ & \\
\hline
\end{tabular}

ricanos a quienes consideran los exponentes de la IC. O sea, más de la mitad citan a los representantes de la TF, destacando Anselm Strauss, Barney Glasser y Juliet Corbin. En menor medida aluden a otros como Norman Denzin o Janice Morse. Otro tercio cita a autores europeos y destacan exponentes de la teoría de las representaciones sociales, como Serge Moscovici y Denise Jodelet; luego están quienes desarrollan programas de cómputo, como Thomas Muhr, creador del programa Atlas-Ti; y en menor medida autores españoles de obras generales sobre la IC, entre los que destacan Gregorio Rodríguez y José Ignacio Olabuenaga. Mención aparte merecen las escasas referencias a la obra de autores latinoamericanos que trabajan en el campo. Por lo tanto, comúnmente se cita a los autores anglosajones, luego a integrantes del grupo al que pertenece el primer autor, y en raras ocasio- nes a académicos del país o la región, entre los cuales se encuentran Eduardo Menéndez para el caso de México y María Cecilia Minayo para el de Brasil.

Vistos en conjunto, los trabajos revisados presentan una serie de limitaciones y ausencias en las estrategias metodológicas empleadas. Aquí aludimos al diseño, el muestreo, la obtención de la información, el uso de programas de cómputo y el análisis. El 62\% de los trabajos no explicitan el diseño empleado ni las estrategias utilizadas; por lo común suelen presentarse como de naturaleza cualitativa. Otro 38\% emplea estrategias definidas, o sea, $14 \%$ son de corte etnográfico, $4 \%$ estudios de caso y $20 \%$ se autodenominan como descriptivos o interpretativos. Por otro lado, 63\% explicitan el tipo de muestreo empleado. De éstos, la mitad hace uso del muestreo por conveniencia, $24 \%$ el propositivo, $20 \%$ 
el bola de nieve y los restantes el teórico y el aleatorio sistemático.

Las estrategias de obtención de la información utilizadas son de índole diversa, aunque predominan las entrevistas en sus diversas modalidades. También se emplean la observación participante y la no participante, los listados libres, las notas de campo e incluso los cuestionarios. Sin embargo predomina el uso de las entrevistas ya que $88 \%$ de los trabajos dependen de ellas y una proporción menor de la observación $(28 \%)$. Cabe destacar que $50 \%$ de los estudios empíricos refiere haber utilizado la triangulación metodológica.

Los programas de cómputo son ampliamente usados en los trabajos revisados, y casi la mitad $(48 \%)$ reporta la utilización de uno de ellos. Los más empleados son el Atlas Ti y el Ethnograph (19 y 15\% respectivamente). Sólo unos cuantos investigadores hacen uso de los programas QRS Nud*ist, Anthropac, Kwalitan y Alceste 45.

Por otra parte, $79 \%$ menciona haber llevado a cabo un determinado análisis cualitativo de los datos, de los cuales $40 \%$ alude a la teoría fundamentada y $23 \%$ al análisis de contenido. Otros tipos de análisis como el fenomenológico, el hermenéutico y del discurso son empleados muy raras veces. Sin embargo, la mayoría no explicita los procedimientos empleados pues se limita a señalar que han realizado determinado análisis cualitativo de los datos; incluso algunos refieren haberlo llevado a cabo mediante un programa de cómputo. ${ }^{37}$

Por otra parte, $66 \%$ de los trabajos empíricos revisados formulan propuestas a partir de los resultados encontrados, por lo general combinando dos o más. Sin embargo, la mayoría de tales propuestas suelen ser vagas y de poco contenido. Por ejemplo, 56\% de ellos recomiendan educar y capacitar más a los profesionales de la salud y a la población en general; otro 30\% a realizar más investigaciones sobre el tema; 28\% a utilizar los resultados encontrados, mientras que $21 \%$ a impulsar más programas de prevención e intervención. Sin embargo, tales propuestas adolecen de información básica sobre cómo habrán de implementarse, quién(es) estará(n) a cargo, las estrategias a emplear y los recursos necesarios, entre otras cosas.

Buen número de estos trabajos aparece en revistas nacionales e internacionales con reconocimiento académico. No obstante, su impacto científico es bajo, sobre todo al valorársele en función del FI de las revistas y el número de veces que son citados. Casi la mitad de las revistas $(46 \%)$ donde se publican estos trabajos no tienen FI, 24\% tienen uno menor a 1.000 y solo 30\% 1.000 y más. En el cuadro III podemos observar que el promedio de citas de los trabajos revisados fue de $4.6 \mathrm{y}$ que varía según diversos factores. Las mujeres publican

\section{Cuadro III \\ Promedio de citas de los trabajos SEGÚN DiverSOS FACTORES. MÉXICO, 2000-2009. Colaboración Y PUBLICACIÓN}

Promedio citas

\begin{tabular}{ll}
$\begin{array}{l}\text { Sexo del primer autor principal } \\
\text { Hombre }\end{array}$ & 5.5 \\
\hline Mujer & 4.3
\end{tabular}

Tipo de colaboración

Internacional $\quad 6.8$

\begin{tabular}{ll} 
Nacional & 5.0 \\
\hline Institucional & 4.0 \\
\hline Sin colaboración & 0.4
\end{tabular}

País de revista

\begin{tabular}{ll} 
Reino Unido & 10.1 \\
\hline EUA & 5.45 \\
\hline Brasil & 4.8 \\
\hline México & 4.1 \\
\hline Otros Latinoamérica & 0.5 \\
& \\
Idioma & \\
Inglés & 6.5 \\
\hline Español & 4.1
\end{tabular}

Fl de revista

\begin{tabular}{lc}
$>2.000$ & $\mathrm{II} .0$ \\
\hline $\mathrm{I} .00 \mathrm{I}-2.000$ & 5.2 \\
\hline $0.00 \mathrm{I}-\mathrm{I} .000$ & 4.0 \\
\hline $\operatorname{Sin} \mathrm{FI}$ & 0.6
\end{tabular}

Total

4.6

más en este campo, pero los hombres son más citados, al igual que los trabajos realizados con académicos extranjeros, los publicados en revistas anglosajonas, en inglés, y sobre todo en función del FI de la revista. Así, la probabilidad de ser citado es casi 20 veces mayor en aquellos publicados en una revista con FI superior a 2.000 que en una revista sin FI.

Finalmente, en el cuadro IV se puede constatar lo anterior al observar los diez autores más citados en esta revisión. Como se dijo, la mayoría estaban adscritos al Instituto Nacional de Salud Pública y la Universidad de Guadalajara. Tales trabajos fueron publicados a principios de la década analizada, en revistas inglesas y norteamericanas, así como en Salud Pública de México (SPM), siendo el FI de todas las revistas superior a 2.000, con la excepción de SPM que en ese momento tenía 1.101. 


\section{Cuadro IV}

Diez trabajos más citados, autores, Revistas y su FI. México, 2000-2009

\begin{tabular}{|c|c|c|c|c|}
\hline Num. & Autor & Num. citas* & Revista & $\mathrm{Fl}$ \\
\hline I & Bronfman $\mathrm{M}$, et al. ${ }^{38}$ & 35 & AIDS & 4.020 \\
\hline 2 & Hijar M, et al..$^{39}$ & 29 & Soc Sci Med & 3.588 \\
\hline 3 & Castro $R$ et al..$^{40}$ & 25 & J Women's Health Gend Based Med & 1.770 \\
\hline 4 & Nigenda G, et al..$^{4 l}$ & 25 & BMC Public Health & 2.220 \\
\hline 5 & Pelcastre-Villafuerte B, et al..$^{35}$ & 20 & Salud Publica Mex & 1.101 \\
\hline 6 & Villaseñor-Farias M, et al. ${ }^{33}$ & 20 & Salud Publica Mex & 1.101 \\
\hline 7 & Stern C, et al. ${ }^{42}$ & 20 & Salud Publica Mex & 1.101 \\
\hline 8 & Álvarez-Gordillo G, et al. ${ }^{43}$ & 18 & Salud Publica Mex & 1.101 \\
\hline 9 & Mercado-Martínez F, Ramos Herrera. ${ }^{10}$ & 15 & Qual Health Res & 2.261 \\
\hline 10 & Mercado-Martínez F, et al44 & 15 & J Health Commun & 2.434 \\
\hline
\end{tabular}

\section{Discusión}

Este trabajo revisa parte importante de la producción científica sobre la ICS generada en México en la primera década del siglo XXI. Según se expuso, la revisión no incluyó todos los trabajos realizados en el país en este campo debido a una serie de preocupaciones de los autores sobre su calidad, accesibilidad y pertinencia. Aunque menos en cantidad que los excluidos, las publicaciones aquí revisadas son aquellas difundidas en revistas que suelen considerarse de calidad en el medio académico. Su selección también consideró su accesibilidad ante el desafío y dificultades que implicaba encontrar los libros, capítulos y la denominada literatura gris en este campo y a nivel nacional. Otro factor presente en la selección fue el de la frontera entre lo considerado como un tema sanitario; más que adoptar una mirada biomédica, se adoptó por una visión más incluyente capaz de explorar parte del proceso salud-enfermedad-atención.

A principios de la década, Cisneros ${ }^{45}$ reportaba una producción incipiente y marginal de los estudios cualitativos en el área de la salud en el país. A diferencia de tal señalamiento, aquí hemos encontrado un número importante y creciente de trabajos cuya difusión a nivel internacional es notoria al ser casi semejante a la nacional en términos numéricos. Todo ello pareciera formar parte de una tendencia más amplia que refleja la aceptación generalizada de la ICS, no solo en el país sino en la región latinoamericana y a nivel mundial. ${ }^{46}$

Los avances logrados en la década analizada no pueden minimizarse. Por ejemplo, algunos trabajos se han publicado en las mejores revistas en el campo a nivel mundial. Pero ello no impide reconocer un conjunto de situaciones que inciden en la consolidación y el enriquecimiento del mismo campo en México. Una de ellas consiste en la adopción a ultranza y la dependencia de posturas empiristas, particularmente de la teoría fundamentada. Ello se ha venido dando mediante varios mecanismos: al adoptarla como la principal "postura teórica", aun cuando diste de considerarse como una teoría per se, la referencia permanente a sus autores más representativos, su empleo como estrategia en el diseño y el análisis, así como el uso de programas de cómputo sustentados en la misma, como es el Atlas-Ti. Tal hallazgo nos parece debería ser objeto de análisis. Si bien se ha documentado que la TF es una de las estrategias empleadas en la IC en el ámbito internacional en ciertas áreas y disciplinas, ello no significa que sea la más idónea ni la más utilizada en todos los contextos y áreas. Por ejemplo, McKibbon \& $\mathrm{Gaad}^{47}$ reportan haber encontrado más estudios fenomenológicos en su revisión de las publicaciones cualitativas en las revistas clínicas a nivel internacional. El predominio de la TF en nuestro medio contrasta, por otra parte, con la ausencia de otras perspectivas teóricas empleadas, tal como el paradigma crítico y el activista cuya presencia es notoria en la región. Éste último se caracteriza, entre otras cosas, por su oposición a los paradigmas eurocéntricos y su propuesta de construir uno de tipo endógeno, enraizados en las circunstancias propias y capaz de reflejar la compleja realidad en que vive la población. ${ }^{48}$ También destaca la ausencia de corrientes críticas que suelen ser empleadas en campos cercanos, tales como en la medicina social y la salud colectiva latinoamericana. ${ }^{49}$ Incluso, la aplicación de la teoría de las representaciones sociales usada por los psicólogos sociales constituye la excepción más que la regla. Por todo ello, parte importante de la producción 
mexicana revisada en este campo no es teórica y enfatiza lo metodológico e instrumental. En consecuencia, esta misma producción pudiera ser objeto de las críticas que se han hecho a numerosos estudios inspirados en la teoría fundamentada; entre otras, su alejamiento de su raíz interpretativa, su énfasis en la metodología y la técnica más que en los procesos investigados, su descontextualización y su falta de interés por cuestiones como el poder y la desigualdad social. ${ }^{50}$

En relación con las temáticas estudiadas, cabe destacar que asuntos sanitarios de importancia en el país han sido olvidados o minimizados, al igual que ciertos grupos de la población. Por ejemplo, estos estudios no han prestado atención a la formación y actualización de los recursos humanos ni a las percepciones, los significados y las prácticas de los profesionales de la salud, incluyendo las de los tomadores de decisiones; tampoco se han preocupado por las enfermedades trasmisibles, tales como el dengue o el paludismo, cuya presencia ha sido notable a lo largo de la década. Ello sin mencionar determinadas enfermedades crónicas de gran impacto médico-social como la enfermedad renal crónica o las enfermedades respiratorias. De máxima importancia también ha sido la atención a la población urbana adulta, pero a costa de otros sectores y grupos de la población, sobre todo la no urbana. Una pregunta a explorar en el futuro es ¿cuál es el papel de los organismos internacionales en la definición de la agenda de la investigación cualitativa en México?

Además de lo anterior, es posible cuestionar: ¿Cuál ha sido el impacto de la producción revisada? Aquí sólo contamos con elementos para explorar este asunto en la academia. Dos rasgos pueden ser objeto de atención: su impacto marginal y desigual. Marginal en tanto que la mayoría de los trabajos no son citados y/o se publican en revistas sin FI. Incluso, los trabajos más citados, utilizando el criterio empleado, reciben entre 15 y 40 citas, una vez eliminadas las citas de los autores hechas a sí mismos. La diferencia es abismal no sólo al comparar estos trabajos con los de autores anglosajones en el mismo campo, sino incluso con autores de la región, como los brasileños. Por ejemplo, algunos artículos de estos últimos autores son citados más de 100 veces durante el mismo periodo analizado, tal como en el caso de Novaes. ${ }^{51}$ Una de las preguntas a explorar en el futuro es sobre las razones por las cuales estos trabajos suelen ser tan poco citados, sobre todo por investigadores del mismo país y que trabajan el mismo tema. En el extremo opuesto estaría explorar la lógica que se emplea en los trabajos más citados en Salud Pública de México, revista con un FI menor al de las otras revistas que se presentan en el cuadro IV. Otra faceta del impacto de los trabajos remite a las propuestas que formulan los autores de estos estudios. Aquí destaca la falta de interés e imaginación de tales propuestas porque, entre otras cosas, es común encontrar sugerencias tan obvias como las de capacitar más a los profesionales de la salud o a los enfermos, así como a investigar más sobre el tema. En este sentido pareciera que no hay diferencia alguna entre estas propuestas y conclusiones respecto a las de los estudios cuantitativos, ya sean epidemiológicos o de servicios de salud.

A pesar del número limitado de citas a los trabajos revisados, las diferencias encontradas en torno a las mismas citas es un hallazgo perturbador. Es decir, el perfil de los trabajos más citados es el de aquéllos elaborados por académicos varones, de una universidad pública o institución de salud importante, con colaboración internacional, publicados en revistas anglosajonas, en inglés y con un FI mayor a 3.000, o menor mientras aparezca en Salud Pública de México. El problema de fondo radica en que pocos trabajos revisados reúnen tal perfil, mientras que lo opuesto pareciera ser la regla. No se han encontraron estudios que examinen los factores aquí revisados en el mismo campo pero en otros contextos. Aun así, los presentes hallazgos coinciden con informes que muestran que los estudios cualitativos suelen ser llevados a cabo más por mujeres ${ }^{52}$ a diferencia de otros campos como el de la salud colectiva en el cual predominan los hombres. ${ }^{49}$

Varias iniciativas y estrategias podrían impulsarse para fortalecer y consolidar la ICS en México. Una es el establecimiento de redes de investigadores y grupos de trabajo de diversas instituciones de salud interesadas en el campo. Un tópico de máximo interés sería comenzar a escuchar las múltiples voces y preocupaciones de los investigadores involucrados. Otra vía sería impulsar la creación de repositorios con la producción mexicana en ICS a fin de darla a conocer entre los investigadores de este campo y favorecer la crítica, discusión y reconocimiento de los trabajos realizados en el país y la región. Un tercer tema sería fortalecer y vincular las iniciativas de formación de recursos humanos que han aparecido a lo largo del país en este campo. Prioridad semejante tendría el hecho de fortalecer la capacitación teórica de jóvenes investigadores para impulsar trabajos de mayor solidez teórico-conceptual, a la vez que se recupera la tradición crítica que caracteriza parte de la producción en salud, no sólo en México sino en la región latinoamericana.

Por último, se ha seleccionado y revisado parte de la producción mexicana en materia de ICS. Entre otros temas a explorar en el futuro está el de verificar si un panorama semejante ocurre en otras áreas con una fuerte tradición en la IC, como en educación, agricultura o desarrollo rural. Incluso, sería de enorme interés comparar si tendencias semejantes ocurren en otros países de la región, tal como en Brasil, por hacer referencia al 
país líder en este campo en la región. Por último, una y otra vez nos preguntamos ¿qué tan diferente será la producción científica mexicana en este campo difundida por otros medios a los aquí revisados?

\section{Agradecimientos}

Agradecemos al Mtro. César Padilla Altamira por sus comentarios y sugerencias. También a los evaluadores anónimos por sus valiosos comentarios y propuestas. Y a la Dra. Ciara Kierans por su apoyo en la traducción del resumen.

Declaración de conflicto de intereses: Los autores declararon no tener conflicto de intereses.

\section{Referencias}

I. Mercado FJ, Gastaldo D, Calderón C. Investigación cualitativa en salud en Iberoamérica: Métodos, análisis y ética. Guadalajara: Universidad de Guadalajara/ Servicio Vasco de Salud Osakidetza, 2002.

2. Gastaldo D, Mercado-Martínez FJ, Ramasco-Gutierrez M, Lizardi-Gomez A, Gil-Nebot MÁ. Qualitative health research in Ibero-America: the current state of the science. J Transcult Nurs 2002;13(2):90-108.

3. Mercado-Martínez F. Investigación cualitativa en América Latina: Perpectivas críticas en salud. Intl J Qual Methods 2002;I(I).

4. Amezcua M, Carricondo A. Investigación cualitativa en España: Análisis de la producción bibliográfica en salud. Index Enferm 2000;28(29):26-34. 5. Romero M, Rodríguez E, Durand A, Aguilera RM. Veinticinco años de investigación cualitativa en salud mental y adicciones con poblaciones ocultas. Primera parte. Salud Mental 2003;26(6):76-83.

6. Fuentes M, López $S$. La investigación social en salud: comunicaciones recientes en SPM. Salud Publica Mex 2005;47(I):5-7.

7. Szasz I, Lerner S. Para comprender la subjetividad: Investigación cualitativa en salud reproductiva y sexualidad. México: El Colegio de México, 1996.

8. Harzing AWK, Van der Wai R. Google Scholar as a new source for citation analysis. Ethics Sci Environ Polit 2008;8(I):6I-73.

9. Weber R. Basic content analysis. London: Sage, 1990.

10. Granados-Cosme J, Torres-Cruz C, Delgado-Sánchez G. La vivencia del rechazo en homosexuales universitarios de la Ciudad de México y situaciones de riesgo para VIH/SIDA. Salud Publica Mex 2009;5I (6):482-488. II. Hernández-Rosete D, García O, Bernal E, Castañeda-Camey X, Lemp G. Migración y ruralización del SIDA: relatos de vulnerabilidad en comunidades indígenas de México. Rev Saude Publica 2008;42(I):I3I-I 38. 12. Castro R, Erviti J. Las redes sociales en la experiencia del aborto: un estudio de casos con mujeres de Cuernavaca (México). Estud Sociol 2003;2I (63):585-6II.

13. Flores-Palacios F, Leyva R. Representación social del SIDA en estudiantes de la Ciudad de México. Salud Publica Mex 2003;45(supl 5):S624-S63I.

14. Mercado-Martínez F, Ramos-Herrera I. Diabetes: The layperson's theories of causality. Qual Health Res 2002;12(6):792-806.

15. López-Amador K, Ocampo-Barrio P. Creencias sobre su enfermedad, hábitos de alimentación, actividad física y tratamiento en un grupo de diabéticos mexicanos. Arch Med Fam 2007;19(2):80-86.

16. Lavielle P, Clark P, Martínez H, Mercado F, Ryan G. Conducta del enfermo ante el dolor crónico. Salud Publica Mex 2008;50(2):147-I54.
17. García S, Yam E, Firestone M. "No party hat, no party": successful condom use in sex work in México and the Dominican Republic. Reprod Health Matters 2006; I4(28):53-62.

18. Nuño-Gutiérrez B, González C. La representación social que orienta las decisiones paternas al afrontar el consumo de drogas de sus hijos. Salud Publica Mex 2004;46: I23-131.

19. Zabicky G, Solis L. El juramento: maniobra no médica coadyuvante en el manejo de los sujetos con consumo patológico de etanol de México. Aproximación inicial. Salud Mental 2000;23(4):22-27.

20. Wagner F, González C, Aguilera R, Ramos-Lira L, Medina-Mora $M$, Anthony J. Oportunidades de exposición al uso de drogas entre estudiantes de secundaria de la Ciudad de México. Salud Mental 2003;26(2):22-32.

21. Nigenda G, Ruiz-Larios JR, Bejarano-Arias R, Alcalde-Rabanal J, BonillaFernández P. Análisis de las alternativas de los migrantes mexicanos en Estados Unidos en América para atender sus problemas de salud. Salud Publica Mex 2009;5I(5):407-4I6.

22. Herrera C, Campero L, Caballero M, Kendall T. Relación entre médicos y pacientes con $\mathrm{VIH}$ : influencia en apego terapéutico y calidad de vida. Rev Saude Publica 2008;42(2):249-255.

23. Hernández-Tezoquipa I, Arenas-Monreal L, Valdez-Santiago R. "No dejarse caer en cama": las mujeres y los servicios de salud. Cad Saude Publica 2005;2I(4):I210-12I6.

24. Herrera-Kiengelher L, Villamil-Álvarez M, Pelcastre-Villafuerte B, CanoValleeguih F, López-Cervantes M. Relación del personal de salud con los pacientes en la Ciudad de México. Rev Saude Publica 2009;43(4):589-594. 25. Torres-López T, Acosta D, Aguilar M. Entre médicos y pacientes buenos y malos: puntos de vista de los actores de la relación médico-pacientes. Invest Salud 2004;6(I): I4-2I.

26. Mora J, Flores-Palacios F, Alba MD, Marroquín M. Construcción de significados acerca de la salud mental en población adulta de una comunidad urbana marginal. Salud Mental 2003;26(5):5I-60.

27. Berenzon-Gorn S, Saavedra-Solano N, Alanis-Navarro S. Estrategias utilizadas por un grupo de mujeres mexicanas para cuidar su salud emocional: autoatención y apoyo social. Salud Publica Mex 2009;5I(6):474-48I

28. Cabello M, Zuñiga J. Aspectos interpersonales y familiares asociados a la obesidad: un análisis fenomenológico. Ciencia UANL 2007;10(2):I83-188. 29. Pérez S, Díez-Urdanivia S. Estudios sobre alimentación y nutrición en México: una mirada a través del género. Salud Publica Mex 2007;49:445-453. 30. Herrera-Suárez C, Alba JG-D, Vásquez E, Romero E, Romo H, Troyo R. Consenso cultural sobre alimentos en adolescentes embarazadas de Guadalajara, México. Rev Salud Publica 2008;10(5):723-731.

3I. Valdez-Santiago R, Arenas-Monreal L, Hernández-Tezoquipa I. Experiencia de las parteras en la identificación de mujeres maltratadas durante el embarazo. Salud Publica Mex 2004;46:56-63.

32. Herrera $C$, Agoff $C$. Dilemas del personal médico ante la violencia de pareja en México. Cad Saude Publica 2006;22(I I):2349-2357.

33. Villaseñor-Farias M, Castañeda-Torres J. Masculinidad, sexualidad, poder y violencia: análisis de significados en adolescentes. Salud Publica Mex 2003;45(supl I):S44-S57.

34. Hernández I, Arenas L, Valdez R. El cuidado a la salud en el ámbito doméstico: interacción social y vida cotidiana. Rev Saude Publica 200I;35(4):443-450.

35. Abrantes-Pego R, Arjonilla S. Descentralización del sector de salud y conflictos con el gremio médico en México. Rev Saúde Pública 2002;36(3): 324-329.

36. Treviño-Siller S, Pelcastre-Villafuerte B, Márquez-Serrano M. Experiencias de envejecimiento en el México rural. Salud Publica Mex 2006;48:30-38.

37. Zarco A, Mora G, Pelcastre-Villafuerte B, Flores M, Bronfman M. Aceptabilidad de los suplementos alimenticios del programa oportunidades. Salud Publica Mex 2006;48:325-331. 
38. Bronfman M, Leyva R, Negroni M, Rueda C. Mobile populations and HIVIAIDS in Central America and Mexico: research for action. AIDS 2002; 16(suppl 3):S42-S49.

39. Hijar M, Trostle J, Bronfman M. Pedestrian injuries in Mexico: a multimethod approach. Soc Sci \& Med 2003;57:2149-2159.

40. Castro R, Campero L, Hernández B, Langer A. A study on maternal mortality in Mexico through a qualitative approach. J Women's Health \& Gend Based Med 2000;9(6):679-690.

4I. Nigenda G, Langer A, Kuchaisit C, Romero M, Rojas G, Al-Osimy M, et al. Womens' opinions on antenatal care in developing countries: results of a study in Cuba, Thailand, Saudi Arabia and Argentina. BMC Public Health 2003;3.

42. Stern C, Fuentes-Zurita C, Lozano-Trevoño L. Masculinidad y salud sexual y reproductiva: un estudio de caso con adolescentes de la Ciudad de México. Salud Publica Mex 2003;45(supl I):S34-S43.

43. Álvarez-Gordillo G, Álvarez-Gordillo J, Dorantes-Jiménez J, HalperinFrisch D. Percepciones y prácticas relacionadas con la tuberculosis y la adherencia al tratamiento en Chiapas, México. Salud Publica Mex 2000;42:520-528.

44. Mercado FJ, Robles L, Moreno N, Franco C. Inconsistent Journalism: The coverage of chronic diseases in the Mexican press. J Health Commun 2001; 6:235-247
45. Cisneros C. Qualitative social research in Mexico. Forum Qual Soc Res 2000; I (I).

46. Turato ER. Métodos qualitativos e quantitativos na área da saúde: definições, diferenças e seus objetos de pesquisa. Rev Saude Publica 2005;39(3):507-5।4.

47. McKibbon KA, Gadd CS. A quantitative analysis of qualitative studies in clinical journals for the 2000 publishing year. BMC Med Inform Decision Making 2004;4(I):II.

48. Mora-Osejo LE, Fals Borda O. A superação do eurocentrismo. Enriquecimento do saber sistêmico e endógeno sobre o nosso contexto tropical. En: Santos B. Conhecimento prodente para uma vida decente. Sao Paulo: Cortez Editora, 2003:71 I-720.

49. Nunes ED. A trajetória das ciências sociais em saúde na América Latina: revisão da produção científica. Rev Saude Publica 2006;40(Esp):64-72. 50. Bryant A, Charmaz K. The Sage Handbook of Grounded Theory. Thousand Oaks: Sage, 2002.

5I. Novaes HMD. Avaliação de programas, serviços e tecnologias em saúde. Rev Saude Publica 2000;34:547.

52. Grant $L$, Ward KB, Rong $X L$. Is there an association between gender and methods in sociological research? Am Sociol Rev 1987;52(6):856-862. 